\title{
RELATÓRIO DE AVALIAÇÃO DO II ENCONTRO DE PESQUISA EM HISTÓRIA DA EDUCAÇÃO E CULTURA ESCOLAR: ARQUIVOS, ACERVOS E MUSEUS
}

\section{Realização:}

GT de História da Educação ANPUH/RS

\section{Organização:}

Profa. Dra. Doris Bittencourt Almeida (UFRGS)

Prof. Dr. José Edimar de Souza (UCS)

Prof. Dndo. Eduardo Cristiano Hass da Silva (UNISINOS)

Profa. Dnda. Bárbara Virgínia Groff da Silva (PUCRS)

Local: Faculdade de Educação da UFRGS - (Sala 102)

Data: 05 e 06 de julho de 2017

Horário: 17:30 - 21:00

Nos dias 5 e 6 de julho de 2017, nas dependências da Faculdade de Educação da Universidade Federal do Rio Grande do Sul, realizou-se o II Encontro de Pesquisa em História da Educação e Cultura Escolar, com o tema "Arquivos, Acervos e Museus". O encontro reuniu pesquisadores, professores, profissionais e estudantes interessados pelo tema elencado.

O presente relatório visa fazer um balanço geral do encontro, destacando a importância de dar continuidade a esta iniciativa, bem como a de promover publicações que destaquem os diferentes espaços para pesquisa, bem como alguns cuidados teóricos e metodológicos.

\section{PROGRAMAÇÃo}

\section{$1^{\circ}$ Dia - o5 de julho de 2017 (17:30 - 21:00)}

Arquivos, Acervos e Museus para Pesquisa em História da Educação: Europa, América Latina e Brasil.

Mediação: Eduardo Cristiano Hass da Silva (Dndo. Unisinos, Secretário GT de História da Educação da ANPUH/RS). 
(17:30) Abertura

> (17:45 - 18:25) Pesquisa em História da Educação e Cultura Escolar: A importância dos acervos - Prof. Dra. Maria Helena Camara Bastos (PUCRS)

$>$ (18:25 - 18:50) Espaços de pesquisa, histórias e memórias: transfigurações e possibilidades em contexto ibero-americano - Tatiane de Freitas Ermel (Dra. em Educação pela PUCRS)

$>$ (18:50 - 19:15) Manuais Pedagógicos em História da Educação: Acervos Portugueses - Fernando Ripe (Dndo. em Educação pela UFPEL);

(19:15 - 19:35) Coffee

$>$ (19:35 - 20:00) Vestígios da cultura escrita escolar: a pesquisa em arquivos e acervos (Brasil e França) - Carolina Monteiro (UFRGS);

$>$ (20:00 - 20:25) Museus em História da Educação: O Museu Histórico Nacional do Rio de Janeiro - Ana Carolina Gelmini De Faria (UFRGS); (20:25 - 21:00) Debate/ Perguntas

\section{$2^{\circ}$ Dia - o6 de julho de 2017 (17:30 - 21:00)}

Acervos de Espaços Escolares e Não Escolares para Pesquisa em História da Educação. Prof. Dra. Doris Bittencourt Almeida (Mediação: Eduardo Hass)

\section{(17:30) Recados/ Comunicações}

(17:40 - 18:05) Arquivo Morto? Possibilidades de Pesquisa nos Acervos da Escola Pública: O Colégio Cândido José Godói e um estudo do PIBID. - Bárbara da Silva (Dnda em Educação pela PUCRS)

(18:05 - 18:35) O Arquivo da FACED para além da documentação oficial: Memórias orais e escritas ordinárias como possibilidades de pesquisa (2010-2017) - Dra. Doris Bittencourt Almeida (UFRGS) e Lucas Costa Grimaldi (Me. em Educação pela UFRGS)

$>$ (18:35 - 19:00) Uma instituição escolar: diversas possibilidades de acervos - a experiência de pesquisa do Colégio Nossa Senhora de Lourdes, Farroupilha/RS - Gisele Belusso (Dnda UCS);

(19:00 - 19:20) Coffee

$>$ (19:20 - 19:45) Acervos e Memoriais Escolares: O Memorial do Colégio Farroupilha de Porto Alegre/RS - Alice Rigoni Jacques (Dra. em Educação pela PUCRS, Memorial do Colégio Farroupilha)

$>$ (19:45 - 20:10) Lares Arquivados: A Memória Estudantil Universitária nos Acervos das Casas de Estudante Autônomas de Porto Alegre-RS (1964-1985) - Marcos Luiz Hinterholz (Mdo. em Educação pela UFRGS); (20:10 - 20:40) Debate/ Perguntas (20:40 - 21:00) Entrega dos certificados 
2. LEVANTAMENTO DAS INSTITUIÇÕES DOS PARTICIPANTES

\subsection{Comissão Organizadora}

\begin{tabular}{|l|c|}
\hline Instituição & Quantidade \\
\hline Universidade Federal do Rio Grande do Sul & $\mathbf{2}$ \\
\hline Pontifícia Universidade Católica do Rio Grande do Sul & $\mathbf{1}$ \\
\hline Universidade de Caxias do Sul & $\mathbf{1}$ \\
\hline Universidade do Vale do Rio dos Sinos & $\mathbf{1}$ \\
\hline Total: & $\mathbf{5}$ \\
\hline
\end{tabular}

\subsection{Palestrantes e mediador}

\begin{tabular}{|l|c|}
\hline Instituição & Quantidade \\
\hline Universidade Federal do Rio Grande do Sul & 5 \\
\hline Pontifícia Universidade Católica do Rio Grande do Sul & 3 \\
\hline Universidade Federal de Pelotas & $\mathbf{1}$ \\
\hline Memorial do Colégio Farroupilha de Porto Alegre & $\mathbf{1}$ \\
\hline Universidade de Caxias do Sul & $\mathbf{1}$ \\
\hline Universidade do Vale do Rio dos Sinos & $\mathbf{1}$ \\
\hline Total: & $\mathbf{1 2}$ \\
\hline
\end{tabular}

\subsection{Ouvintes}

\begin{tabular}{|l|c|}
\hline Instituição & Quantidade \\
\hline Universidade Federal do Rio Grande do Sul & 25 \\
\hline CEUE - Pré-Vestibular & 11 \\
\hline Pontifícia Universidade Católica do Rio Grande do Sul & 7 \\
\hline Universidade do Vale do Rio dos Sinos & 2 \\
\hline Unilassale & 2 \\
\hline Universidade Luterana do Brasil & 1 \\
\hline SEDUC & 1 \\
\hline Instituto Federal de Educação & 1 \\
\hline Rede Pública de Ensino & 1 \\
\hline Rede Privada de Ensino & 1 \\
\hline Objetivo Pré-Vestibular & 1 \\
\hline COREM & 1 \\
\hline
\end{tabular}




\begin{tabular}{|l|c|}
\hline Fundação de Estatística do Estado do Rio Grande do Sul & 1 \\
\hline CESUCA & 1 \\
\hline Instituto Histórico geográfico do Rio Grande do Sul & 1 \\
\hline Total: & $\mathbf{5 7}$ \\
\hline
\end{tabular}

\subsection{Monitores}

\begin{tabular}{|l|c|}
\hline Instituição & Quantidade \\
\hline Universidade Federal do Rio Grande do Sul & 2 \\
\hline Pontifícia Universidade Católica do Rio Grande do Sul & $\mathbf{1}$ \\
\hline Total: & $\mathbf{3}$ \\
\hline
\end{tabular}

\subsection{Total de envolvidos}

\begin{tabular}{|l|c|}
\hline Função & Quantidade \\
\hline Comissão Organizadora & 1 \\
\hline Palestrantes e mediador & 12 \\
\hline Ouvintes & 57 \\
\hline Monitores & 3 \\
\hline Total: & $\mathbf{7 3}$ \\
\hline
\end{tabular}

\subsection{Total de envolvidos por instituição}

\begin{tabular}{|l|c|}
\hline Instituição & Quantidade \\
\hline Universidade Federal do Rio Grande do Sul & 32 \\
\hline CEUE - Pré-Vestibular & 11 \\
\hline Pontifícia Universidade Católica do Rio Grande do Sul & 11 \\
\hline Universidade do Vale do Rio dos Sinos & 3 \\
\hline Unilassale & 2 \\
\hline Universidade de Caxias do Sul & 2 \\
\hline Universidade Luterana do Brasil & 1 \\
\hline SEDUC & 1 \\
\hline Instituto Federal de Educação & 1 \\
\hline Rede Pública de Ensino & 1 \\
\hline Rede Privada de Ensino & 1 \\
\hline Objetivo Pré-Vestibular & 1 \\
\hline
\end{tabular}

${ }^{1} \mathrm{O}$ total de envolvidos na Comissão Organizadora é de cinco pessoas, no entanto, quatro destes membros estão entre palestrantes e mediadores. Dessa forma, para a base de cálculo do total de envolvidos, utilizaremos a quantidade de um. 


\begin{tabular}{|l|c|}
\hline COREM & 1 \\
\hline Fundação de Estatística do Estado do Rio Grande do Sul & 1 \\
\hline CESUCA & 1 \\
\hline Instituto Histórico geográfico do Rio Grande do Sul & 1 \\
\hline Universidade Federal de Pelotas & 1 \\
\hline Memorial do Colégio Farroupilha de Porto Alegre & 1 \\
\hline Total: & $\mathbf{7 3}$ \\
\hline
\end{tabular}

\subsection{Avaliação de ouvintes}

\begin{tabular}{|c|c|c|c|c|c|c|}
\hline & Ótimo & $\begin{array}{l}\text { Muito } \\
\text { bom }\end{array}$ & Bom & Ruim & $\begin{array}{l}\text { Muito } \\
\text { ruim }\end{array}$ & $\begin{array}{l}\text { Não que- } \\
\text { ro/posso } \\
\text { opinar }\end{array}$ \\
\hline $\begin{array}{l}\text { 1. Como você avalia } \\
\text { a organização do } \\
\text { evento? }\end{array}$ & 10 & 4 & - & - & - & - \\
\hline $\begin{array}{l}\text { 2. Com relação à } \\
\text { temática central do } \\
\text { evento "Pesquisa em } \\
\text { História da Educa- } \\
\text { ção e Cultura Esco- } \\
\text { lar: Arquivos, Acer- } \\
\text { vos e Museus", você } \\
\text { avalia que o encon- } \\
\text { tro foi: }\end{array}$ & 9 & 5 & - & - & - & - \\
\hline $\begin{array}{l}\text { 3. Com relação à } \\
\text { coerência entre ob- } \\
\text { jetivos estabelecidos } \\
\text { para o encontro e a } \\
\text { sua realização, você } \\
\text { avalia que foi de } \\
\text { maneira geral: }\end{array}$ & 8 & 6 & - & - & - & - \\
\hline $\begin{array}{l}\text { 4. De forma geral, } \\
\text { como você avalia os } \\
\text { conteúdos das pa- } \\
\text { lestras realizadas } \\
\text { durante o evento? }\end{array}$ & 10 & 4 & - & - & - & - \\
\hline
\end{tabular}

